\title{
Überzeugungen von Grundschullehrkräften zum Umgang mit nicht-deutschen Erstsprachen im Unterricht
}

\author{
Sarah Désirée Lange $\mathbb{D}$ - Sanna Pohlmann-Rother
}

Angenommen: 6. April 2020 / Online publiziert: 27. April 2020

(C) Der/die Autor(en) 2020

Zusammenfassung Mit der vorliegenden Studie werden die Überzeugungen von berufstätigen Grundschullehrkräfte zum Umgang mit nicht-deutschen Erstsprachen im Unterricht untersucht und die Bedeutung formaler und informeller bzw. nonformaler Lerngelegenheiten für diese Überzeugungen analysiert. Die Ergebnisse der Fragebogenstudie $(N=123)$ zeigen, dass die Mehrheit der befragten Lehrkräfte davon überzeugt ist, dass Erstsprachen zumindest zeitweise einen Platz in ihrem Unterricht haben sollten. So stimmen beispielsweise $75 \%$ der befragten Lehrkräfte der Aussage mindestens teilweise zu, dass die Schülerinnen und Schüler von Zeit zu Zeit Lerninhalte in ihren Erstsprachen besprechen dürfen. Dem Einsatz von nichtdeutschen Lernmaterialien im Unterricht stehen knapp $60 \%$ der Lehrkräfte mindestens positiv gegenüber. Die multiplen Regressionsanalysen zeigen, dass sich die erstsprachenintegrierenden Überzeugungen anhand der Nutzung von formalen und informellen bzw. non-formalen Lerngelegenheiten teilweise erklären lassen. Eine formale Aus- und Weiterbildung im Themenbereich des Deutschen als Zweitsprache hat einen positiven Einfluss auf die Überzeugungen von Grundschullehrkräften zum Einbezug von Erstsprachen in ihren Unterricht. Zudem sind es auch informelle bzw. non-formale Sprachkontakte (wie etwa Alltagskommunikation oder Mediennutzung in einer Fremdsprache), deren Nutzung die Überzeugungen der Grundschullehrkräfte hinsichtlich des Umgangs mit Erstsprachen im Unterricht beeinflussen.

Schlüsselwörter Grundschule · Mehrsprachigkeit · Lehrkräfte · Überzeugungen · Familiensprachen

Dr. S. D. Lange $(\bowtie) \cdot$ Prof. Dr. S. Pohlmann-Rother

Lehrstuhl für Grundschulpädagogik und Grundschuldidaktik, Fakultät für Humanwissenschaften,

Institut für Pädagogik, Julius-Maximilians-Universität Würzburg, Würzburg, Deutschland

E-Mail: Sarah.Lange@uni-wuerzburg.de

Prof. Dr. S. Pohlmann-Rother

E-Mail: Sanna.Pohlmann-Rother@uni-wuerzburg.de 


\title{
Beliefs of primary school teachers on dealing with non-German first languages in teaching
}

\begin{abstract}
This study examines the beliefs of in-service primary school teachers regarding the use of non-German first languages in teaching. In addition, the study focuses what relevance formal and informal resp. non-formal learning opportunities have for these beliefs. The results of the questionnaire survey $(N=123)$ show that the majority of teachers interviewed believe that first languages should have a place in their lessons, at least temporarily. $75 \%$ of the teachers, for example, agree at least in part with the statement that students from time to time are allowed to discuss learning content in their first language. Almost $60 \%$ of the teachers have a positive attitude towards the use of non-German learning material in class. The multiple regression analyses show that beliefs in favour of including first languages in teaching can be partially explained by the use of formal and informal or nonformal learning opportunities. Formal education and training in the subject area of German as a second language has a positive influence on the beliefs of primary school teachers to include first languages in teaching. Also, the use of informal or non-formal language contacts (such as everyday communication or media use in a foreign language) influence the beliefs of primary school teachers regarding the use of first languages in class.
\end{abstract}

Keywords Primary School $\cdot$ Multilingualism $\cdot$ Teachers $\cdot$ Beliefs $\cdot$ Family languages

\section{Themenkontext: Mehrsprachigkeit als Ressource in der Grundschule}

Aufgrund globalisierungsbedingter Veränderungen der Gesellschaft (wie etwa durch Migrations- und Fluchtbewegungen) ist eine durch Mehrsprachigkeit geprägte Klassengemeinschaft Normalität in deutschen Grundschulen (Braun et al. 2018). Laut dem Bildungsbericht von 2018 haben etwa 30\% der Kinder in Kindertageseinrichtungen im Alter von drei Jahren bis zum Schuleintritt einen Migrationshintergrund (im Jahr 2017). Von diesen Kindern wachsen etwa $66 \%$ mit einer anderen Familiensprache als Deutsch auf (Autorengruppe Bildungsberichterstattung 2018, S. 284). Die Frage nach dem Umgang mit migrationsbedingter Mehrsprachigkeit in Schule und Unterricht wird im wissenschaftlichen Diskurs nicht ausschließlich auf der Grundlage empirischer Erkenntnisse geführt, sondern ist auch durch normative Setzungen und Entscheidungen geprägt (Gogolin 2017). Kennzeichnend für diesen Diskurs ist in den letzten Jahren ein Perspektivenwechsel von einer bis in die frühen 1980er Jahre hinein dominierenden „Defizitperspektive“ (Gogolin und KrügerPotratz 2010, S. 173) hin zu einem ressourcenorientierten Blick auf Lernchancen für alle Schülerinnen und Schüler durch den Einbezug nicht-deutscher Erstsprachen. Während vor über 20 Jahren Gogolin und Neumann (1997) zu dem Ergebnis kamen, dass die im schulischen Alltag vorzufindende Mehrsprachigkeit häufig als von der Norm abweichend wahrgenommen wird, zeigen auch aktuelle Studien, dass ein Verbot der Erstsprachen von Lehrkräften als Hilfestellung in der Entwicklung hin 
zu sprachlicher ,Normalität‘ verstanden wird (Steinbach 2017, S. 84). Dies verweist auf die Persistenz monolingualer Muster im schulischen Denk- und Handlungsraum (Huxel 2016, S. 184). Die Forderung nach der Berücksichtigung nicht-deutscher Erstsprachen von Schülerinnen und Schülern im Unterricht impliziert eine Abkehr von dem im deutschen Schulsystem vorherrschenden ,monolingualen Habitus“ (Gogolin 1994) und setzt entsprechende Überzeugungen der Lehrkräfte voraus. Für die Frage, ob Lehrkräfte die Mehrsprachigkeit ihrer Schülerinnen und Schüler als Chance oder als Herausforderung für schulische Bildungsprozesse wahrnehmen und in der Folge die unterschiedlichen Erstsprachen in den Unterricht einbeziehen oder ausschließen, erscheinen die Überzeugungen der Lehrkräfte relevant, weil diesen als Facette pädagogischer Professionalität im kompetenztheoretischen Diskurs der Professionsforschung eine handlungsleitende Funktion zugeschrieben wird (Baumert und Kunter 2006).

\section{Theoretischer Hintergrund}

\subsection{Erstsprachen im Grundschulunterricht}

In diesem Beitrag wird mit ,Erstsprachen im Unterricht“ der didaktische Einbezug von nicht-deutschen Erstsprachen in den deutschsprachigen Regelunterricht verstanden. Fallstudien an Grundschulen zeigen, dass Lehrkräfte auf der Ebene der Einzelschule über große Handlungsspielräume im Umgang mit Mehrsprachigkeit verfügen (z. B. Fürstenau 2013). Aber ob und wenn ja, in welcher Intensität und auf welche Art und Weise die Erstsprachen von Schülerinnen und Schülern einen Platz im Unterricht haben sollten, wird kontrovers diskutiert (Gogolin 2017). Der Umgang mit Erstsprachen im Unterricht wird aufgrund der Interdisziplinarität des Themas Mehrsprachigkeit - als individuelles wie auch gesellschaftliches Phänomen - linguistisch, soziologisch wie auch pädagogisch verhandelt (Tracy 2014).

Während psycholinguistische Studien kognitive Vorteile durch Mehrsprachigkeit hervorheben (Bialystok 2005) und in der Soziolinguistik die sprachliche Flexibilität durch Code-Switching auch im Rahmen des Schulunterrichts näher betrachtet wird (Auer 1999), werden aus soziologischer Perspektive insbesondere die Mechanismen und sozialen Bedingungen des Spracherwerbs im Kontext von Migration, Integration und Bildungserfolg in den Blick genommen (Esser 2006). Dabei wird in Bezug auf die ,time-on-task-Hypothese“ angenommen, dass der zusätzliche unterrichtliche Zeitaufwand für das Lernen in der Herkunftssprache auf Kosten des Erlernens der Unterrichtssprache gehe. Aktuelle Analysen anhand von PISA-2012-Daten belegen jedoch, dass es keinen Anlass für die Vermutung gibt, dass die familiäre Nutzung der Herkunftssprachen die Aneignung schulisch relevanter Kompetenzen behindere (Rauch 2019). Der aktuelle Forschungsstand bietet eher Hinweise auf positive Effekte des Einbezugs der Erstsprachen in den Unterricht bspw. auf ein lernförderliches Schulklima (im Überblick: Gogolin 2017).

Aus pädagogischer Sicht stellt sich die Frage des Umgangs mit migrationsgesellschaftlicher Differenz; dazu lassen sich verschiedene pädagogische Orientierungen unterscheiden - darunter die stärker handlungsbezogenen Konzepte der Ausländer- 
pädagogik und klassischen Interkulturellen Pädagogik. Die Kritik an der Ausländerpädagogik richtet sich dabei auf die defizitorientierten Forderungen nach Assimilation an die Mehrheitsgesellschaft. An Ansätzen der Interkulturellen Pädagogik wird kritisiert, dass die Betonung von Differenzen zur Verstärkung von Gruppendichotomisierungen und zu Zuschreibungen führen kann (vgl. Hummrich und Terstegen 2020, S. 16-23). Dagegen werden in neueren Ansätzen wie der von Mecheril (2016) geprägten Migrationspädagogik auch gesellschaftlich-systematische sowie macht- und rassismuskritische Dimensionen miteinbezogen. Grundlegend ist dabei die Reflexion der kollektiven Zugehörigkeiten von Individuen, deren Komplexität erst unter intersektionaler Betrachtung angemessen beschrieben werden kann (vgl. Nohl 2014, S. 125-135).

Aus grundschulpädagogischer Perspektive ist danach zu fragen, wie kindgemäße Lernumgebungen im Bewusstsein der unterschiedlichen Lernvoraussetzungen und unter Einbezug der lebensweltlichen Mehrsprachigkeit zu gestalten sind. Dieser Anspruch lässt sich mit den Prinzipien des grundschulpädagogischen Selbstverständnisses begründen (Fürstenau 2017). Dazu zählen der demokratische Auftrag der Grundschule als ,Schule der Vielfalt und Gemeinsamkeit" (Schorch 2007, S. 35) zur Gesellschafts- und Bildungsteilhabe, die Berücksichtigung der kindlichen Lebenswelt im Unterricht, die Initiierung individueller und sozialer Lernprozesse sowie die Unterstützung der Persönlichkeitsentwicklung.

Didaktische Funktionen Welche Begründungslinien können für die Nutzung von Erstsprachen im Grundschulunterricht herangezogen werden? Folgend wird ein Systematisierungsversuch für didaktische Funktionen angeboten: 1) Eine soziale Funktion ist denkbar, indem die Mehrsprachigkeit im Klassenverband wertgeschätzt und für Gruppenbildungsprozesse genutzt wird (bspw. Fürstenau 2017, S. 20) (z. B. gruppenstärkende Aktivitäten, Wertschätzung innerhalb der Klasse sowie Außendarstellung wie Klassenzimmergestaltung). Zudem kann die Einbindung nicht-deutscher Erstsprachen 2) eine identitätsstiftende Funktion einnehmen (Krumm 2009, S. 238-239), wenn die Sprachkompetenzen von Schülerinnen und Schülern gezielt in den Unterricht einbezogen werden, um bspw. das Bewusstsein für verschiedene sprachliche Identitäten zu fördern (z.B. Reflexion von Perspektivenwechsel und Kommunikationssituationen). 3) Auch kann der Berücksichtigung nicht-deutscher Erstsprachen eine spracherwerbsstützende Funktion zukommen, wenn es mehrsprachigen Schülerinnen und Schülern ermöglicht wird, durch Sprachvergleiche ihre Erstsprachenkenntnisse für den Erwerb der deutschen Sprache zu nutzen (Akbulut et al. 2017, S. 72). Ausgehend von der Interdependenzhypothese (Cummins 1979), werden mit der Sprachennutzung fachliche Vorteile für die mehrsprachigen Kinder erwartet (z. B. Gogolin 2010, S. 541). 4) Eine bildende Funktion ist denkbar hinsichtlich früher informeller Kontakte mit Mehrsprachigkeit, um die Reflexion von Normalitätsvorstellungen im Umgang mit allochthonen Sprachen wie auch das Bewusstsein für eine globalisierte und durch Vielfalt geprägte Gesellschaft anzuregen (vgl. Globales Lernen: Lange 2017). 5) Darüber hinaus kann die Ressource Mehrsprachigkeit gezielt für das fachliche Lernen genutzt werden (fachliche Funktion), bspw. zur Erarbeitung mathematischer Zugänge durch den metakognitiven Nutzen der Erstsprachen (Meyer und Prediger 2011, S. 201). In Anlehnung an den kompetenztheoretischen Diskurs 
zur Lehrkräfteprofessionalität kann angenommen werden, dass die Überzeugungen auch für unterrichtliches Handeln bedeutsam sind. Vor diesem Hintergrund wird der Frage nachgegangen, welche Überzeugungen Grundschullehrkräfte hinsichtlich des Einbezugs nicht-deutscher Erstsprachen in den Unterricht aufweisen.

\subsection{Berufsbezogene Überzeugungen von Grundschullehrkräften}

\subsubsection{Kompetenztheoretische Verortung von Überzeugungen im Professionalisierungsdiskurs}

Das einflussreiche Modell zum kompetenztheoretischen Verständnis von professioneller Kompetenz nach Baumert und Kunter (2006) unterscheidet die vier Kompetenzfacetten Professionswissen, Motivationale Orientierungen, Selbstregulation und Überzeugungen. Es wird angenommen, dass Überzeugungen die Wahrnehmung der Welt strukturieren und filtern (Pajares 1992). Berufsbezogene Überzeugungen gelten als relevant für die Steuerung und Begründung von unterrichtlichem Handeln (Liegmann 2012). Wie die Studie von Aksoy (2015) jedoch zeigt, ist der Zusammenhang zwischen den Überzeugungen und dem Handeln von Lehrkräften höchst komplex und geprägt von Übereinstimmungen und Widersprüchen.

Der Begriff der Überzeugungen wurde lange als ,Containerbegriff‘ für das Mentale von Individuen genutzt und gleichgesetzt mit einer Vielzahl von anderen Konstrukten (bspw. Einstellungen) (Trautmann 2005, S. 39). In den letzten Jahren lassen sich Bemühungen beobachten, das Konzept der Überzeugungen für und durch empirische Operationalisierungen theoretisch präziser greifbar zu machen. Nach Skott (2015, S. 18-20) lassen sich bei allen Unklarheiten die folgenden vier Kernaspekte benennen: Überzeugungen (1) sind individuelle mentale Konstrukte; (2) bestehen aus miteinander verwobenen kognitiven und affektiven Anteilen; (3) zeichnen sich durch zeitliche und kontextunabhängige Stabilität aus; (4) beeinflussen, wie Lehrkräfte ihren Alltag wahrnehmen und interpretieren. Im Prozess der Reformierung der Lehrkräftebildung wurde in den letzten Jahren der Fokus auf die Ausbildung des Professions- und Reflexionswissens gelegt und der Bereich der Überzeugungen vernachlässigt. So wird der Großteil der Studien zu den Überzeugungen von Lehramtsstudierenden durchgeführt mit der Erwartung, Erkenntnisse zur Verbesserung der Lehrkräfteausbildung zu erhalten. Der Blick auf Grundschullehrkräfte in der Praxis wurde bislang vernachlässigt (Oser und Blömeke 2012). Im Unterschied zu Studierenden erleben aber nur Lehrkräfte die Auseinandersetzung mit der Praxis und machen entsprechend intensive Erfahrungen im Umgang mit einer sprachlich-heterogenen Schülerschaft; es kann angenommen werden, dass diese die Überzeugungen der Lehrkräfte beeinflussen.

\subsection{2 Überzeugungen von Grundschullehrkräften zum Umgang mit Erstsprachen}

In der Grundschulpädagogik stellt die empirische Erfassung des Konzepts der Überzeugungen zum Umgang mit Erstsprachen von Grundschullehrkräften ein Forschungsdesiderat dar. Überzeugungen sind immer auf eine bestimmte berufliche Anforderung hin ausgerichtet (vgl. Reusser et al. 2011, S. 642). Zum Umgang 
mit migrationsbedingter Heterogenität liegen im deutschsprachigen Raum einzelne Studien vor (z. B. Edelmann 2006; Hachfeld 2013). Es fehlt jedoch an Untersuchungen, die explizit den Umgang mit Mehrsprachigkeit im Unterricht fokussieren und berufstätige Grundschullehrkräfte und deren Überzeugungen in den Blick nehmen. Vorliegende Studien thematisieren entweder nicht spezifisch das Konstrukt der Überzeugungen (Maak et al. 2015), nicht spezifisch das Thema Mehrsprachigkeit (Wischmeier 2012), untersuchen als Zielgruppe Lehramtsstudierende (Fischer und Ehmke 2019; Hammer et al. 2016) oder ErzieherInnen (Kratzmann et al. 2017) jedoch nicht Grundschullehrkräfte.

Kratzmann et al. (2017) untersuchen die Einstellungen von ErzieherInnen zum Umgang mit Erstsprachen im Rahmen einer Fortbildung und können dabei die folgenden drei Dimensionen identifizieren: multilingual-pädagogische, kompensatorische und assimilatorische Dimension. Den Items der kompensatorischen Dimension wurde dabei am stärksten zugestimmt $(M=4,66 ; 1=$ gar nicht; $5=$ völlig $)$, gefolgt von der Zustimmung zu den Items der multilingual-pädagogischen $(M=4,12)$ und der assimilatorischen Dimension $(M=3,43)$.

Methodisch hervorzuheben sind die von Fischer und Ehmke (2019) entwickelten Skalen zur Erfassung der Überzeugungen zu sprachlich-kultureller Heterogenität bei Lehramtsstudierenden, die Ausgangspunkt der vorliegenden Adaptionen für die Zielgruppe der Grundschullehrkräfte war.

\subsubsection{Formale und informelle bzw. non-formale Lerngelegenheiten zum Umgang mit Erstsprachen}

Es liegen umfangreiche Studien dazu vor, dass allgemeine Faktoren - darunter Alter, Geschlecht (z. B. Özer et al. 2013) oder auch die Persönlichkeit (Bernat und Gvozdenko 2005) - Überzeugungen beeinflussen. Zudem implizieren Forschungsbefunde, dass zur Aufklärung von Überzeugungen bei Lehrkräften Unterrichtserfahrungen und persönliche Ressourcen, wie z.B. Selbstwirksamkeit (Hellmich und Görel 2014; Ottley et al. 2015), relevant sind. Während Fischer und Ehmke (2019) bei Lehramtsstudierenden zeigen können, dass sich die Überzeugungen der Studierenden zu sprachlich-kultureller Heterogenität in hohem Maße durch die Häufigkeit von besuchten Lehrveranstaltungen im Studium erklären lassen, ist wenig dazu bekannt, ob und wenn ja, welche Lerngelegenheiten die Überzeugungen von berufstätigen Lehrkräften beeinflussen. In der BLUME-Studie zeigte sich, dass sich die Mehrheit der Grundschullehrkräfte weder im Studium noch in Fort- und Weiterbildungen intensiv mit der Förderung von mehrsprachigen Kindern beschäftigte (PohlmannRother und Lange 2020). Zwar lassen sich in einzelnen Bundesländern in den letzten Jahren bildungspolitische Entwicklungen hinsichtlich der institutionellen Verankerung des Themas Mehrsprachigkeit in der Lehrkräfteausbildung beobachten, etwa die verpflichtende Umsetzung des $\mathrm{DaZ}^{1}$-Moduls in Nordrhein-Westfalen (Putjata et al. 2016). Jedoch bleibt dabei bislang offen, was die Studierenden davon für ihren Beruf lernen.

\footnotetext{
1 DaZ steht für Deutsch als Zweitsprache.
} 


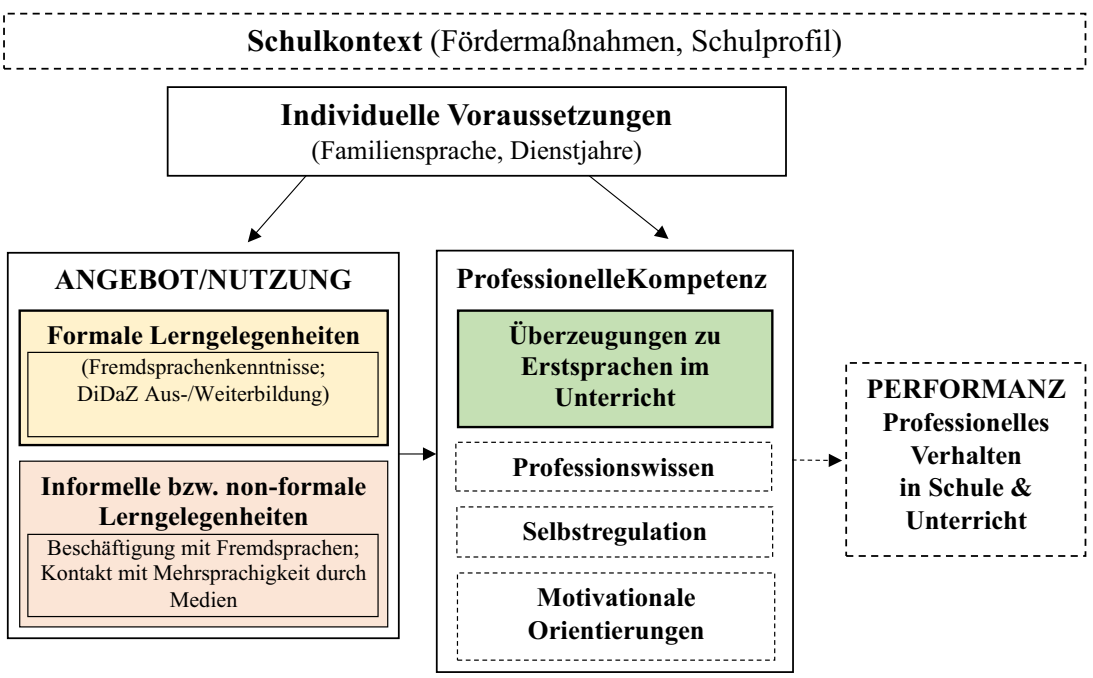

Abb. 1 Theoretisches Modell zur Bedeutung von Lerngelegenheiten für die Überzeugungen von Lehrkräften. (Eigenentwicklung, basierend auf Kunter et al. 2011, S. 59)

Nach dem Modell von Kunter et al. (2011, S. 59) spielen Lerngelegenheiten für die Genese von Überzeugungen als Professionalisierungsfacette von Lehrkräften eine relevante Rolle. Für die vorliegende Studie wurde das Modell erweitert, indem Lerngelegenheiten in formale und informelle bzw. non-formale Lerngelegenheiten unterschieden wurden (vgl. Abb. 1). Nach Baumbast et al. (2012) ist formales Lernen ,organisiert und strukturiert, findet in formalisierten Bildungseinrichtungen statt und führt im Allgemeinen zur Zertifizierung“ (ebd., S. 12); informelles Lernen erfolgt unbeabsichtigt und meistens unbewusst. Es ist nicht auf ein bestimmtes Feld begrenzt, sondern kann überall geschehen (ebd., S. 20); non-formales Lernen ,,findet außerhalb von formalen Bildungseinrichtungen statt, wobei meistens damit außerschulisch gemeint ist, [es] wird üblicherweise nicht zertifiziert, kann aber u. U. zur Zertifizierung führen, wobei diese Zertifizierung keine weitergehende Bildungsberechtigung impliziert, ist weniger strukturiert als formales Lernen“ (ebd., S. 15). Hervorgehoben wird in der Diskussion jedoch die Komplexität der Wechselwirkungen zwischen formalen und informellen Lernprozessen (Brodowski 2012), welche eine quantitativ-empirische Erfassung sowie die Abgrenzung von non-formalen zu formalen oder informellen Lerngelegenheiten erschwert.

Biedermann et al. (2012) konnten einen signifikanten Zusammenhang zwischen den (retrospektiv eingeschätzten) Lerngelegenheiten und den Überzeugungen zum Erwerb mathematischen Wissens nachweisen und schlussfolgern daraus, dass Lernangebote berufsbezogene Überzeugungen von Lehramtsstudierenden beeinflussen können. Bouley et al. (2015) konnten den Einfluss von universitären und außeruniversitären Lerngelegenheiten auf das Fachwissen und das fachdidaktische Wissen von angehenden Lehrkräften aufzeigen. Bezogen auf das Thema Mehrsprachigkeit deuten die Ergebnisse der BLUME-Studie darauf hin, dass Lehrkräfte, die sich mit dem Thema Mehrsprachigkeit in der Ausbildung beschäftigten, eher der Überzeu- 
gung sind, angemessen auf den Umgang mit Mehrsprachigkeit vorbereitet zu sein (vgl. Pohlmann-Rother und Lange 2020). Dieses Ergebnis steht im Einklang mit Ergebnissen der DaZKom²-Studie, die auf den geringen Stellenwert der Nutzung von DaZ-bezogenen Lerngelegenheiten im Lehramtsstudium verweisen (vgl. Ehmke und Lemmrich 2018). Für die hier vorgestellte Studie wird ein besonderes Augenmerk auf die Nutzung informeller und non-formaler Lerngelegenheiten gelegt - ein bislang wenig untersuchter Aspekt (vgl. Tachtsoglou und König 2017, S. 308).

\subsection{Fragestellungen}

Ausgehend von den zuvor beschriebenen Forschungsdesiderata lassen sich die folgenden Forschungsfragen ableiten, die mit der vorliegenden Studie beantwortet werden:

1. Welche berufsbezogenen Überzeugungen haben Grundschullehrkräfte hinsichtlich des Umgangs mit Erstsprachen im Unterricht?

2. Welche Bedeutung haben formale und informelle bzw. non-formale Lerngelegenheiten für die Überzeugungen von Grundschullehrkräften zum Umgang mit Erstsprachen im Unterricht?

\section{Datengrundlage und methodisches Vorgehen}

\subsection{Stichprobe}

Die Daten zur Beantwortung der formulierten Fragestellungen wurden anhand eines Onlinefragebogens erhoben, der getestete Skalen wie auch Eigenentwicklungen umfasst. Der Fragebogen wurde zunächst mit $N=12$ kognitiven Pretestinterviews (Willis 2005) pilotiert und dann im Zeitraum von Mai bis Juni 2018 eingesetzt. Insgesamt nahmen $N=123$ Grundschullehrkräfte aus einem Schulamtsbezirk in Bayern an der Befragung teil. Die Kriterien für das Sampling waren die aktuelle Anstellung als Grundschullehrkraft und das Innehaben einer Klassenleitung - Lehrkräfte in Pension, im Mutterschutz oder in Abordnung sind nicht miteinbezogen.

Die Stichprobe beinhaltet mehrheitlich weibliche Lehrkräfte (91\%). Die Grundschullehrkräfte sind durchschnittlich 42 Jahre alt $(\operatorname{Min}=25, \operatorname{Max}=64, S D=10,56)$ und waren zum Erhebungszeitpunkt im Mittel bereits 15,24 Jahre als Lehrkraft an Grundschulen tätig (Min=1, Max=39, SD=10,27). Der überwiegende Teil der Lehrkräfte gibt an, in Deutschland geboren zu sein (98\%). Ähnlich verhält es sich mit den Eltern der Befragten - lediglich $4 \%$ haben eine Mutter und $3 \%$ einen Vater, die/der nicht in Deutschland geboren wurde. Etwas höher ist mit 8,9\% der Anteil der Grundschullehrkräfte, die angeben, dass in ihrer Familie andere Familiensprachen als Deutsch gesprochen werden. Nur eine Person berichtete, zusätzlich zur Erstsprache keine weitere Fremdsprache (bspw. im schulischen Kontext) gelernt zu haben

\footnotetext{
${ }^{2}$ DaZkom steht für Lehrerkompetenz im Bereich Deutsch als Zweitsprache (vgl. https://www.dazkom. de/).
} 
$(8,9 \%=$ eine weitere Sprache gelernt, $40,6 \%=$ zwei weitere Sprachen, 30,7\%=drei weitere Sprachen, $15,8 \%=$ vier weitere Sprachen, $3 \%=$ fünf weitere Sprachen).

\subsection{Fragebogenerhebung}

Für den Fragebogen wurden getestete Skalen für die Zielgruppe der Grundschullehrkräfte adaptiert und relevante Hintergrundvariablen sowie Lerngelegenheiten der Lehrkräfte bezüglich des Themas Mehrsprachigkeit erfasst (vgl. Tab. 2).

\subsubsection{Abhängige Variable}

Überzeugungen zum Umgang mit Erstsprachen (ES) im Unterricht Zur Erfassung der Überzeugungen zum Umgang mit Erstsprachen im Unterricht wurde eine Skala von Fischer und Ehmke (2019), die theoriebasiert für Lehramtsstudierende entwickelt wurde, für die Zielgruppe berufstätiger Grundschullehrkräfte adaptiert (Fischer 2020). Die Grundschullehrkräfte gaben ihre Zustimmung zu insgesamt sieben Aussagen auf einer sechsstufigen Likert-Skala. Die abgefragten Items (Tab. 1) erfassen die Überzeugungen der Lehrkräfte dahingehend, ob im Unterricht auch nichtdeutsche Sprachen sowie Lernmaterial in anderen Sprachen als Deutsch erlaubt ist oder inwiefern verschiedene Sprachen das Lernen des Deutschen erschweren könnten (von $1=$,stimme überhaupt nicht zu“ bis $6=$,stimme voll und ganz zu“). Um die Eignung der adaptierten Skala zu prüfen, wurde eine explorative Faktorenanalyse durchgeführt. Alle Item-Item-Korrelationen zeigen signifikante oder hochsignifikante Korrelationen und der Kaiser-Meyer-Olkin-Test bestätigt die Eignung der Daten für eine Faktorenanalyse (KMO-Index=0,83). Die Faktorenanalyse bestätigte die einfaktorielle Datenstruktur (Hauptkomponentenanalyse, Varimax-Rotation, Eigenwert Komponente $1=50 \%$ ). Die aus einem Hauptfaktor bestehende Skala weist eine hohe interne Konsistenz auf (Cronbachs $\alpha=0,84$ ).

Tab. 1 Itemformulierungen der Skala „Überzeugungen zum Umgang mit Erstsprachen im Unterricht“. (Antwortformat: $1=$,stimme überhaupt nicht $\mathrm{zu}$ “ bis $6=$,stimme voll und ganz zu“)

\begin{tabular}{lll}
\hline Item & Polung & Itemformulierung \\
\hline 1 & + & $\begin{array}{l}\text { Mehrsprachige Lernende sollten im Unterricht die Möglichkeit haben, sich } \\
\text { von Zeit zu Zeit in ihrer Erstsprache auszutauschen }\end{array}$ \\
2 & - & $\begin{array}{l}\text { Wenn Kinder im Unterricht auch andere Sprachen als Deutsch sprechen, dann } \\
\text { leidet das Klassenklima darunter }\end{array}$ \\
3 & - & $\begin{array}{l}\text { Im Unterricht sollten mehrsprachige SchülerInnen ausschließlich Deutsch } \\
\text { sprechen, da sie ein Sprachwechsel irritieren könnte }\end{array}$ \\
4 & + & $\begin{array}{l}\text { Im Unterricht sollten SchülerInnen von Zeit zu Zeit Lerninhalte in ihren Erst- } \\
\text { sprachen besprechen dürfen }\end{array}$ \\
5 & - & $\begin{array}{l}\text { In der Schule sollte nur die deutsche Sprache erlaubt sein, damit mehrsprachi- } \\
\text { ge Kinder leichter Deutsch lernen } \\
6\end{array}$ \\
& - & $\begin{array}{l}\text { Wenn mehrsprachige SchülerInnen im Unterricht ihre Erstsprachen sprechen } \\
\text { dürfen, verliert die Lehrkraft die Kontrolle }\end{array}$ \\
& & $\begin{array}{l}\text { Im Unterricht sollten mehrsprachige Kinder auch Lernmaterialien (z. B. Bü- } \\
\text { cher) in ihren Erstsprachen nutzen dürfen }\end{array}$ \\
\hline
\end{tabular}




\subsubsection{Unabhängige Variablen (Eigenentwicklungen)}

Formale Lerngelegenheiten Fremdsprachenkenntnisse: Die Grundschullehrkräfte wurden nach ihren Fremdsprachenkenntnissen gefragt, die in der Regel im Rahmen von formaler Bildung einen individuellen Kontakt mit Mehrsprachigkeit darstellen („Haben Sie (zusätzlich zu Ihrer/Ihren Erstsprachen) noch weitere Sprachen gelernt (z.B. in der Schule, VHB-Kurs ...)?“; 1= nein; 2=eine weitere Sprache; 3=zwei weitere Sprachen; 4=drei weitere Sprachen; 5=vier weitere Sprachen; $6=$ fünf weitere Sprachen).

DiDaZ 3 -Aus-/Weiterbildung: Um die formale DiDaZ-Ausbildung der Grundschullehrkräfte zu erfassen, wurde gefragt, ob die Lehrkräfte eine (zusätzliche) DiDaZAusbildung absolvierten (dichotomes Antwortformat). Um die DiDaZ-Weiterbildung abzufragen, wurde erhoben, ob sich die Lehrkräfte zum Thema Mehrsprachigkeit selbst weiterbildeten (dichotomes Antwortformat). Aus den beiden Variablen zur DiDaZ-Aus- und Weiterbildung wurde ein additiver Index gebildet und für alle Lehrkräfte berechnet.

Informelle bzw. non-formale Lerngelegenheiten Aufgrund der konzeptionellen Nähe und den entsprechenden Wechselwirkungen (vgl. Baumbast et al. 2012) wurden informelle und non-formale Lerngelegenheiten nicht getrennt erfasst.

Bedeutung des Kontakts mit Fremdsprachen: Die befragten Grundschullehrkräfte wurden gebeten, die Bedeutung der Auseinandersetzung mit Fremdsprachen (auch im Ausland) zu bewerten (,Wie wichtig ist es Ihnen, sich (zu Hause oder im Ausland) mit Fremdsprachen auseinanderzusetzen?"; a) Sprachkurs zum Erwerb einer Fremdsprache; b) Alltagskommunikation in einer Fremdsprache; c) Mediennutzung in einer Fremdsprache; Antwortformat: $1=$ unwichtig bis $4=$ wichtig; Cronbachs Alpha $=0,57)$.

Erfahrungen mit Mehrsprachigkeit durch Medien: Als weitere mögliche Lerngelegenheit wurde erfasst, ob die Grundschullehrkräfte Erfahrungen mit Mehrsprachigkeit durch Medien haben (,Haben Sie Erfahrungen mit Mehrsprachigkeit durch Medien (z. B. Internet, Tageszeitung, TV)?“; dichotomes Antwortformat).

Individuelle Voraussetzungen Dienstjahre: Um die Unterrichtserfahrung zu erfassen, wurden die Dienstjahre als Grundschullehrkraft erhoben.

Familiensprache: Um zu erfassen, ob die Lehrkräfte in der eigenen Familie Kontakt mit Mehrsprachigkeit haben, wurde der Sprachgebrauch in der Familie abgefragt (,Werden in ihrer Familie andere Sprachen als Deutsch gesprochen?“ $0=$ nein; $1=$ ja; bei ,Ja“ wurden auch die jeweiligen Sprachen und die Intensität des Sprachgebrauchs abgefragt) (Tab. 2).

\footnotetext{
${ }^{3}$ Die Studie wurde in Bayern durchgeführt, daher wird die in der Lehramtsprüfungsordnung (LPO) in Bayern verwendete Abkürzung DiDaZ verwendet, die für Didaktik des Deutschen als Zweitsprache steht.
} 
Tab. 2 Stichprobenumfang, Mittelwerte und Standardabweichungen der analysierten Variablen

\begin{tabular}{|c|c|c|c|}
\hline & $n$ & $M$ & $S D$ \\
\hline AV: Überzeugungen Erstsprachen im Unterricht & 123 & 4,03 & 1,12 \\
\hline \multicolumn{4}{|l|}{ UVs: Lerngelegenheiten } \\
\hline \multicolumn{4}{|l|}{ Formelle Lerngelegenheiten } \\
\hline $\begin{array}{l}\text { Fremdsprachenkenntnisse }(1=\text { nein bis } 6=\text { fünf weite }- \\
\text { re Sprachen) }\end{array}$ & 101 & 3,60 & 0,99 \\
\hline DiDaZ-Aus-/Weiterbildung $(0=$ nein; $1=j a)$ & 98 & 0,71 & 0,61 \\
\hline \multicolumn{4}{|l|}{ Informelle bzw. non-formale Lerngelegenheiten } \\
\hline $\begin{array}{l}\text { Bedeutung des Kontakts mit Fremdsprachen } \\
(1=\text { unwichtig bis } 4=\text { wichtig })\end{array}$ & 100 & 2,96 & 0,73 \\
\hline $\begin{array}{l}\text { Erfahrungen mit Mehrsprachigkeit durch Medien } \\
(0=\text { nein; } 1=j a)\end{array}$ & 99 & 0,52 & 0,50 \\
\hline \multicolumn{4}{|l|}{ Individuelle Voraussetzungen } \\
\hline Dienstjahre (offen) & 92 & 15,24 & 10,27 \\
\hline Familiensprache $(0=$ nein $; 1=j a)$ & 101 & 0,09 & 0,29 \\
\hline
\end{tabular}

Unterschiede in der Stichprobengröße kommen durch fehlende Werte zustande

\subsection{Datenauswertung}

Quantitative Auswertung Um der ersten Fragestellung nach der Ausprägung der Überzeugungen der Grundschullehrkräfte zum Umgang mit Erstsprachen nachzugehen, wurden die Daten deskriptiv ausgewertet. Für die zweite Fragestellung nach der Bedeutung der Lerngelegenheiten für die Überzeugungen wurden bivariate Korrelationen berechnet und bei Bedarf Dummyvariablen gebildet. Für die multivariaten Regressionsmodelle wurde aufgrund der kleinen Stichprobengröße ein schmaler Prädiktorensatz ausgewählt. Dabei wurden diejenigen Prädiktoren miteinbezogen, die mit der abhängigen Variable signifikant korrelierten. Um die Bedeutung der formalen und informellen bzw. non-formalen Lerngelegenheiten für die Überzeugungen der Grundschullehrkräfte zum Umgang mit Erstsprachen im Unterricht zu untersuchen, wurden blockweise lineare Regressionsanalysen mit SPSS (Version 24) gerechnet. Modell 1 umfasst die Prädiktoren zu den formalen Lerngelegenheiten, Modell 2 beinhaltet die Prädiktoren zu den informellen bzw. non-formalen Lerngelegenheiten und Modell 3 umfasst die Prädiktoren der formalen und informellen bzw. nonformalen Lerngelegenheiten.

\section{Ergebnisse}

\subsection{Fragestellung 1: Überzeugungen zu Erstsprachen im Unterricht}

Die deskriptive Auswertung zeigt zwar, dass die Frage danach, ob Erstsprachen im Unterricht einen Platz haben (sollten), unter den befragten Grundschullehrkräften insgesamt umstritten ist. Allerdings zeigt der Mittelwert eine deutliche Tendenz hin zu einer positiven Haltung gegenüber dem Einbezug von Erstsprachen $(M=4,03$, $S D=1,12, N=123)$. Zum Vergleich der Verteilungen auf Einzelitemebene sind in 


\section{Überzeugungen zum Umgang mit Erstsprachen im Unterricht}

Im Unterricht sollten mehrsprachige Kinder auch Lernmaterialien (z.B. Bücher) in ihren Erstsprachen nutzen dürfen. (Item $7 \mathrm{M}=3,69 ; \mathrm{SD}=1,69$ )

Im Unterricht sollten mehrsprachige SchülerInnen ausschließlich Deutsch sprechen, da sie ein Sprachwechsel irritieren könnte. (Item 3_M=3,73; SD=1,72)

In der Schule sollte nur die deutsche Sprache erlaubt sein, damit mehrsprachige Kinder leichter Deutsch lernen. (Item 5_M=3,84; $\mathrm{SD}=1,63$ )

Mehrsprachige Lernende sollten im Unterricht die Möglichkeit haben, sich von Zeit zu Zeit in ihrer Erstsprache auszutauschen (Item 1_M=4,11; SD=1,47)

Wenn mehrsprachige SchülerInnen im Unterricht ihre Erstsprachen sprechen dürfen, verliert die Lehrkraft die Kontrolle. (Item 6_M=4,17; SD=1,54)

Im Unterricht sollten SchülerInnen von Zeit zu Zeit Lerninhalte in ihren Erstsprachen besprechen dürfen. (Item $4 \mathrm{M}=4,28 ; \mathrm{SD}=1,40$ )

Wenn Kinder im Unterricht auch andere Sprachen als Deutsch sprechen, dann leidet das Klassenklima darunter. (Item $2 \mathrm{M}=4,37$; $\mathrm{SD}=1,53)$

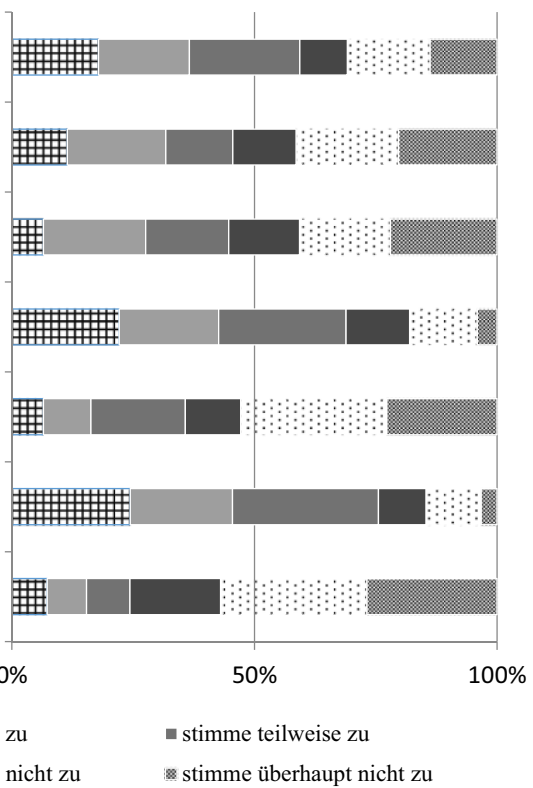

口 stimme voll und ganz zu

- stimme teilweise nicht zu m stimme überwiegend zu
stimme überwiegend nicht zu

Abb. 2 Deskriptive Ergebnisse auf Einzelitemebene der Skala Überzeugungen zum Umgang mit Erstsprachen im Unterricht

Abb. 2 die Mittelwerte aufsteigend dargestellt. Dabei sind die folgenden Aspekte auffallend: Die größte Zustimmung der Lehrkräfte betrifft die beiden Items, in denen es darum geht, dass sich die SchülerInnen mit zeitlicher Einschränkung (,,von Zeit zu Zeit") im Unterricht in ihrer Erstsprache austauschen können (Item 1_stimme voll und ganz $\mathrm{zu}=22,1 \%$ ) und Lerninhalte in ihrer Erstsprache besprechen können (Item 4_stimme voll und ganz zu=22,1\%). Insgesamt zeigen 74,6\% der Lehrkräfte (voll und ganz/überwiegend/teilweise) positiv ausgeprägte Überzeugungen zur lernunterstützenden Funktion von nicht-deutschen Erstsprachen im Unterricht (Item 4). Die höchste Ablehnung zeigen die Lehrkräfte hinsichtlich der Annahme negativer Auswirkungen auf das Klassenklima, wenn nicht-deutsche Erstsprachen im Unterricht gesprochen werden (Item 2_stimme überhaupt nicht zu: 26,8\%). Auch interessant ist, dass 52,9\% der Lehrkräfte angeben, entweder voll und ganz oder überwiegend keine Angst vor dem Kontrollverlust zu haben, wenn andere Erstsprachen im Unterricht gesprochen werden (Item 6). Dem Einsatz von nicht-deutschen Lernmaterialien für mehrsprachige Kinder im Unterricht stehen knapp $60 \%$ der Lehrkräfte (wenn auch nur teilweise/überwiegend) positiv gegenüber (Item 7). 


\subsection{Fragestellung 2: Bedeutung von formalen und informellen bzw. non- formalen Lerngelegenheiten für die Überzeugungen zum Umgang mit Erstsprachen im Unterricht}

Zur Beschreibung des Zusammenhangs der verwendeten Variablen wurden in einem ersten Schritt bivariate Korrelationen berechnet (Tab. 3). Die beiden unabhängigen Variablen UV6 und UV7 korrelieren hoch und signifikant miteinander $\left(\mathrm{r}=0,57^{* *}\right)$. Zwar ist der Toleranzkoeffizient $(T=0,65)$ nicht extrem auffällig, aber aufgrund der hohen Korrelation und der inhaltlichen Nähe der beiden Variablen ist anzunehmen, dass die ,Erfahrungen mit Mehrsprachigkeit durch Medien` zu großen Teilen durch die ,Bedeutung des Kontakts mit Fremdsprachen " mit abgefragt wurden, weshalb die UV7 für die folgenden Regressionsanalysen nicht berücksichtigt wird. Es zeigen sich signifikante Zusammenhänge zwischen den Überzeugungen zu Erstsprachen im Unterricht (AV) und den formalen (Fremdsprachenkenntnisse, DiDaZ-Aus/ Weiterbildung) und informellen bzw. non-formalen Lerngelegenheiten (Bedeutung des Kontakts mit Fremdsprachen).

Zur Beantwortung der zweiten Fragestellung, ob und wenn ja, welche Lerngelegenheiten die Überzeugungen zum Umgang mit Erstsprachen im Unterricht erklären können, wurden Regressionsanalysen gerechnet. Da sich beide Hintergrundvariablen

Tab. 3 Bivariate Korrelationen

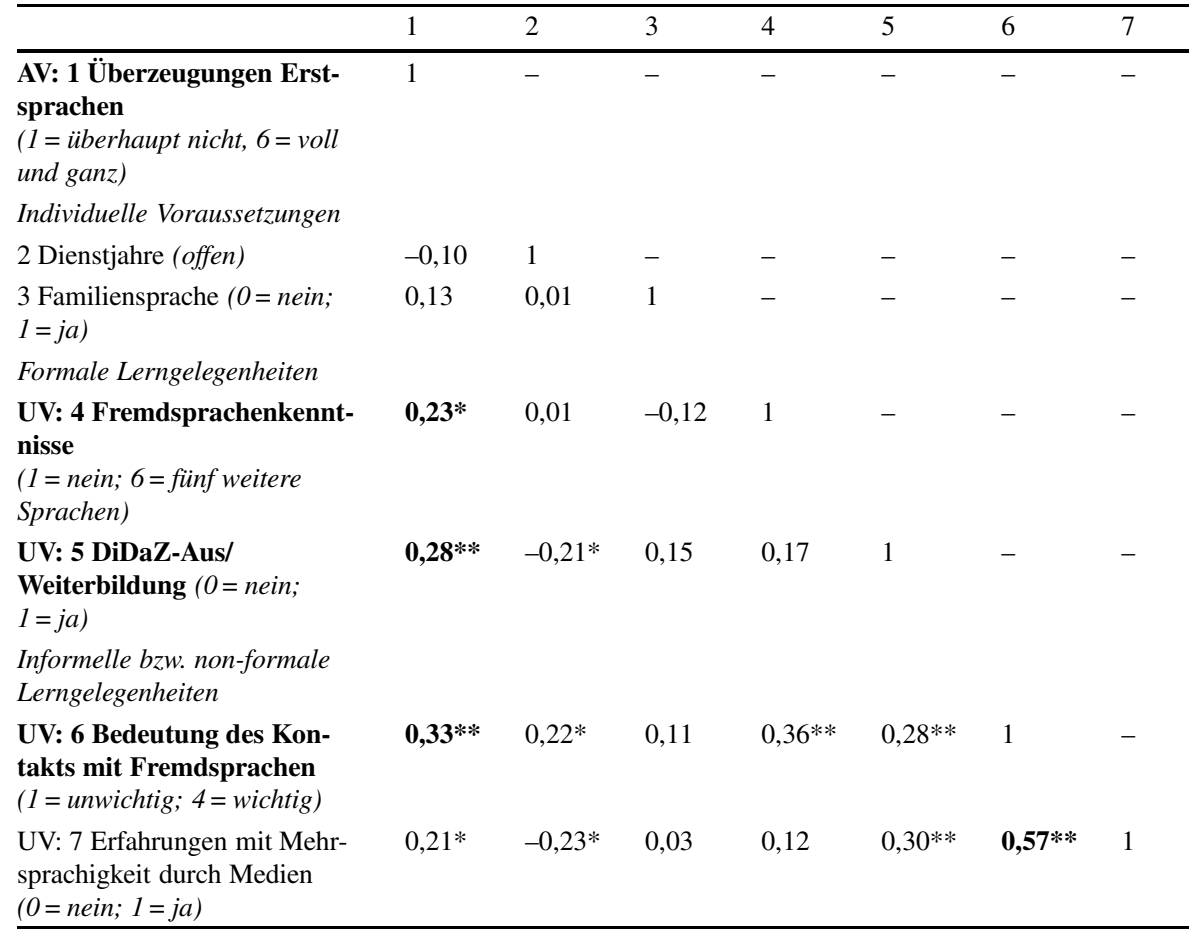

$N=123$

$* p<0,05 ; * * p<0,01 ; * * * p<0,001$ 
Tab. 4 Hierarchische Regressionsanalysen zur Erklärung der Überzeugungen zum Umgang mit Erstsprachen im Unterricht

\begin{tabular}{|c|c|c|c|c|c|c|c|c|c|}
\hline \multirow[t]{2}{*}{ Variablen } & \multicolumn{3}{|c|}{ Modell 1} & \multicolumn{3}{|c|}{ Modell 2} & \multicolumn{3}{|c|}{ Modell 3} \\
\hline & $\beta$ & $B$ & $S E$ & $\beta$ & $B$ & $S E$ & $\beta$ & $B$ & $S E$ \\
\hline \multicolumn{10}{|l|}{$\begin{array}{l}\text { Formale } \\
\text { Lerngelegenheiten }\end{array}$} \\
\hline $\begin{array}{l}\text { Fremdsprachen- } \\
\text { kenntnisse }\end{array}$ & $0,23 *$ & 0,19 & 0,12 & - & - & - & 0,13 & 0,11 & 0,12 \\
\hline $\begin{array}{l}\text { DiDaZ-Aus-/ } \\
\text { Weiterbildungsindex }\end{array}$ & $0,47 *$ & 0,25 & 0,18 & - & - & - & $0,37 *$ & 0,20 & 0,19 \\
\hline \multicolumn{10}{|c|}{$\begin{array}{l}\text { Informelle bzw. non-formale } \\
\text { Lerngelegenheiten }\end{array}$} \\
\hline $\begin{array}{l}\text { Bedeutung des Kon- } \\
\text { takts mit Fremdspra- } \\
\text { chen }\end{array}$ & - & - & - & $\mathbf{0 , 5 1} * *$ & 0,33 & 0,15 & $0,36 *$ & 0,23 & 0,17 \\
\hline $\mathrm{R}^{2}$ & 0,11 & & & 0,11 & & & 0,16 & & \\
\hline
\end{tabular}

$* p<0,05 ; * * p<0,01 ; * * * p<0,001$

(Dienstjahre und Familiensprache) in den bivariaten Korrelationen als nicht relevant erwiesen, wurden diese nicht in die Analysen einbezogen.

Die Ergebnisse der Regressionsanalysen zeichnen folgendes Bild: Je mehr Fremdsprachenkenntnisse und je mehr DiDaZ-bezogene Aus- und Weiterbildungen eine Lehrkraft besucht hat, desto eher dominieren erstsprachenintegrierende Überzeugungen (Tab. 4; Modell 1: Fremdsprachenkenntnisse $\beta=0,23^{*}$; DiDaZ-Aus-und Weiterbildung $\beta=0,47^{*}$ ). Zudem ist die Bedeutung bzw. Wichtigkeit des Kontakts mit Fremdsprachen ein signifikanter Prädiktor für die Vorhersage der Überzeugungen der Lehrkräfte zum Umgang mit Erstsprachen (Tab. 4; Modell 2: Bedeutung des Kontakts mit Fremdsprachen $\left.\beta=0,51^{* *}\right)$. Je wichtiger die Lehrkräfte den Kontakt mit Fremdsprechen beurteilen, desto eher stehen sie der Einbindung der Erstsprachen im Unterricht positiv gegenüber.

Im Gesamtmodell wird wiederum die DiDaZ-Aus- und Weiterbildung sowie die Bedeutung des Kontakts mit Fremdsprachen signifikant, was auf die Relevanz dieser Lerngelegenheiten für die Überzeugungen der Lehrkräfte verweist (Tab. 4; Modell 3: DiDaZ-Aus-und Weiterbildung $\beta=0,37^{*}$; Bedeutung des Kontakts mit Fremdsprachen $\beta=0,36 *)$. Die Varianzaufklärung ist in diesem Modell am höchsten $\left(\mathrm{R}^{2}=0,16\right)$.

\section{Zusammenfassung der Ergebnisse und Diskussion}

Die Ergebnisse zu den Überzeugungen der Grundschullehrkräfte zum Umgang mit Erstsprachen im Unterricht können, vorsichtig positiv ' gewertet werden. Insgesamt überwiegt der Anteil der Lehrkräfte, der davon überzeugt ist, dass Erstsprachen zumindest zeitweise einen Platz in ihrem Unterricht haben sollten. So zeigen die Ergebnisse auf Einzelitemebene eine hohe Zustimmung zur Annahme einer lernunterstützenden Funktion von nicht-deutschen Erstsprachen im Unterricht (Item 4). Interessant ist die hohe Ablehnung gegenüber der Annahme negativer Auswirkungen auf das Klassenklima, wenn nicht-deutsche Erstsprachen im Unterricht gesprochen 
werden (Item 2). Über die Hälfte der Befragten hat entweder voll und ganz oder überwiegend keine Angst vor dem Kontrollverlust, wenn andere Erstsprachen im Unterricht gesprochen werden (Item 6) und befürwortet den Einsatz von nicht-deutschen Lernmaterialien für mehrsprachige Kinder im Unterricht (Item 7).

Die Zusammenschau dieser Ergebnisse zeigt, dass sich der in der normativ geprägten Diskussion um Mehrsprachigkeit geforderte Paradigmenwechsel von einem defizitorientierten Blick zu der Wahrnehmung von Mehrsprachigkeit als ,potenzielle Ressource' teilweise auch in den Überzeugungen der Lehrkräfte widerspiegelt. Die deskriptive Verteilung der Antworten auf Einzelitemebene (Abb. 2) veranschaulicht aber auch die Varianz in den Überzeugungen der Lehrkräfte, in denen sich die ganze Bandbreite an Positionen, die auch in der theoretischen Diskussion (Interdependenz vs. Time-on-task) zu finden ist, nachzeichnen lässt. Aufgrund der komplexen Wechselwirkungen der Zusammenhänge zwischen den Überzeugungen und dem Handeln der Lehrkräfte (Aksoy 2015) erlauben die Daten jedoch keine Schlussfolgerungen zum wertschätzenden Einbezug der nicht-deutschen Erstsprachen auf der Ebene der Unterrichtspraxis. Vorliegende Studien mit ähnlichem Erkenntnisinteresse liegen nur für den Elementarbereich vor, zeigen aber auch tendenziell positive Einstellungen der ErzieherInnen zum Einbezug von Mehrsprachigkeit (multilingual-pädagogische Dimension: $M=4,12 ; 1=$ gar nicht; 5= völlig; Kratzmann et al. 2017).

Die Ergebnisse der Regressionsanalysen der vorliegenden Studie deuten an, dass eine formale Aus- und Weiterbildung im Themenbereich des Deutschen als Zeitsprache (DaZ) einen positiven Einfluss hinsichtlich der Überzeugungen von Grundschullehrkräften zum Einbezug von Erstsprachen in ihren Unterricht haben kann. Dieses Ergebnis kann als Argument einer für alle Grundschullehrkräfte institutionalisierten Beschäftigung mit dem Thema Mehrsprachigkeit in der Lehrkräfteausbildung gesehen werden. Auch die DaZKom-Studie verweist auf signifikante Zusammenhänge zwischen einer DaZ-Ausbildung und den Überzeugungen hinsichtlich der Dimension ,Sprachsensibilität im Fachunterricht ${ }^{\text {- }}$ - jedoch bei Lehramtsstudierenden. Wie in der vorliegenden Studie zeigen sich auch im DaZKom-Projekt keine Zusammenhänge zwischen den Erstsprachen der Studierenden (Deutsch oder nicht-deutsche Erstsprache) und den Überzeugungen zum Umgang mit Mehrsprachigkeit (Hammer et al. 2016). Andererseits scheinen es aber auch informelle bzw. non-formale Sprachkontakte (wie etwa Alltagskommunikation oder Mediennutzung in einer Fremdsprache) zu sein, deren Nutzung die Überzeugungen der Grundschullehrkräfte hinsichtlich des Umgangs mit Erstsprachen im Unterricht beeinflussen.

Limitationen und Ausblick $\mathrm{Ob}$ und inwieweit die Nutzung der formellen und informellen bzw. non-formalen Lerngelegenheiten tatsächlich zur Genese der Überzeugungen der Lehrkräfte beiträgt, kann in der vorliegenden Studie nicht abschließend geklärt werden. Ebenso ist eine umgekehrte Wirkrichtung denkbar, nach der die Überzeugungen der Lehrkräfte die Nutzung von Lerngelegenheiten beeinflussen. Zudem verweisen die bivariaten Korrelationen sowie die Werte der Varianzaufklärung darauf, dass es neben den vorliegend modellierten Einflussgrößen weitere Faktoren gibt, die sich auf die Überzeugungen auswirken. Es ist nicht ausgeschlossen, dass die Antworten der Lehrkräfte auch von unterschiedlichen Verständnissen von Mehr- 
sprachigkeit geprägt sind und diese damit auch die Erfassung der Lerngelegenheiten beeinflussen können.

Der Forschungsgegenstand der berufsbezogenen Überzeugungen ist vielschichtig, komplex und bislang auch methodisch noch nicht zufriedenstellend erfasst. Jedoch kann aus einer kompetenztheoretischen Perspektive angenommen werden, dass die Überzeugungen eine relevante Facette professioneller Kompetenz darstellen, die bedeutsam für einen konstruktiven Umgang mit sprachlicher Heterogenität als Querschnittsaufgabe in der Grundschule ist. Um die Überzeugungen von Lehrkräften zum Umgang mit Mehrsprachigkeit künftig umfassender zu untersuchen, wäre es sinnvoll, Überzeugungen nicht nur quantitativ-empirisch, sondern auch qualitativ zu untersuchen. Dabei wäre es wichtig, entsprechend aktueller pädagogischer Konzepte zu versuchen, auch intersektionale Zusammenhänge sowie kollektive Zugehörigkeiten $\mathrm{zu}$ erfassen. Ein weiteres wichtiges Ziel könnte darin bestehen, die formalen, non-formalen und informellen Lerngelegenheiten detaillierter und präziser voneinander getrennt zu erfassen, um die Überzeugungen von berufstätigen Lehrkräften zum Umgang mit Mehrsprachigkeit weiter aufklären zu können.

Funding Open Access funding provided by Projekt DEAL.

Open Access Dieser Artikel wird unter der Creative Commons Namensnennung 4.0 International Lizenz veröffentlicht, welche die Nutzung, Vervielfältigung, Bearbeitung, Verbreitung und Wiedergabe in jeglichem Medium und Format erlaubt, sofern Sie den/die ursprünglichen Autor(en) und die Quelle ordnungsgemäß nennen, einen Link zur Creative Commons Lizenz beifügen und angeben, ob Änderungen vorgenommen wurden.

Die in diesem Artikel enthaltenen Bilder und sonstiges Drittmaterial unterliegen ebenfalls der genannten Creative Commons Lizenz, sofern sich aus der Abbildungslegende nichts anderes ergibt. Sofern das betreffende Material nicht unter der genannten Creative Commons Lizenz steht und die betreffende Handlung nicht nach gesetzlichen Vorschriften erlaubt ist, ist für die oben aufgeführten Weiterverwendungen des Materials die Einwilligung des jeweiligen Rechteinhabers einzuholen.

Weitere Details zur Lizenz entnehmen Sie bitte der Lizenzinformation auf http://creativecommons.org/ licenses/by/4.0/deed.de.

\section{Literatur}

Akbulut, et al. (2017). Mehrsprachigkeit als Ressource für Sprachbewusstheit. Zeitschrift für Grundschulforschung, 10, 61-74.

Aksoy, K. (2015). What you think is not what you do in the classroom. Procedia-Social and Behavioral Sciences, 199, 675-683.

Auer, P. (1999). From codeswitching via language mixing to fused lects. International Journal of Bilingualism, 3, 309-332.

Autorengruppe Bildungsberichterstattung (2018). Bildung in Deutschland 2018. Ein indikatorengestützter Bericht mit einer Analyse zu Bildung und Migration. Bielefeld: wbv.

Baumbast, S., Hofmann-van de Poll, F., \& Lüders, C. (2012). Non-formale und informelle Lernprozesse in der Kinder- und Jugendarbeit und ihre Nachweise. München: DJI.

Baumert, J., \& Kunter, M. (2006). Stichwort: Professionelle Kompetenz von Lehrkräften. Zeitschrift für Erziehungswissenschaft, 9, 469-520.

Bernat, E., \& Gvozdenko, I. (2005). Beliefs about Language Learning. The Electronic Journal for English as a Second Language (tesl-Ej), 9, 1-21.

Bialystok, E. (2005). Consequences of bilingualism for cognitive development. In J. F. Kroll (Hrsg.), Handbook of bilingualism. Psycholinguistic approaches (S. 417-432). New York: Oxford Press. 
Biedermann, H., Brühwiler, C., \& Krattenmacher, S. (2012). Lernangebote in der Lehrerausbildung und Überzeugungen zum Lehren und Lernen. Beziehungsanalysen bei angehenden Lehrpersonen. Zeitschrift für Pädagogik, 58, 460-475.

Bouley, F., Berger, S., Fritsch, Wuttke, et al. (2015). Der Einfluss von Universitäten und außeruniversitären Lerngelegenheiten auf das Fachwissen und fachdidaktische Wissen von angehenden Lehrkräften an kaufmännisch-berufsbildenden Schulen. Zeitschrift für Pädagogik, Beiheft, 61, 100-115.

Braun, A., Weiß, S., \& Kiel, E. (2018). Interkulturelle Schulentwicklung an Grundschulen. Zeitschrift für Bildungsforschung, 8, 121-135.

Brodowski, M. (2012). Überlegungen zum Zusammenhang formaler und informeller Lernprozesse. Diskurs Kindheits- und Jugendforschung, 7, 431-442.

Cummins, J. (1979). Linguistic interdependence and the educational development of bilingual children. Review of Educational Research, 49, 222-251.

Edelmann, D. (2006). Pädagogische Professionalität im transnationalen sozialen Raum. In C. AllemannGhionda \& E. Terhart (Hrsg.), Kompetenzen und Kompetenzentwicklung von Lehrerinnen und Lehrern (S. 235-249). Weinheim: Beltz.

Ehmke, T., \& Lemmrich, S. (2018). Bedeutung von Lerngelegenheiten für den Erwerb von DaZ-Kompetenz. In T. Ehmke, S. Hammer, A. Köker, U. Ohm \& B. Koch-Priewe (Hrsg.), Professionelle Kompetenzen angehender Lehrkräfte im Bereich Deutsch als Zweitsprache (S. 217-237). Münster: Waxmann.

Esser, H. (2006). Migration, Sprache und Integration. AKI-Forschungsbilanz. https://nbn-resolving.org/ urn:nbn:de:0168-ssoar-113493. Zugegriffen: 19.04.20.

Fischer, N. (2020). Skalendokumentation: Sprachlich-kulturelle Heterogenität in Schule und Unterricht. Frankfurt a.M.: DIPF.

Fischer, N., \& Ehmke, T. (2019). Empirische Erfassung eines „messy constructs“. Zeitschrift für Erziehungswissenschaft, 22, 411-433.

Fürstenau, S. (2013). Schulqualität im Kontext sprachlich-kultureller Heterogenität. In I. Gogolin, I. Lange, U. Michel \& H.H. Reich (Hrsg.), Herausforderung Bildungssprache - und wie man sie meistert (S. 220-238). Münster: Waxmann.

Fürstenau, S. (2017). Migrationsbedingte Mehrsprachigkeit als Gegenstand der Grundschulforschung. Zeitschrift für Grundschulforschung, 10, 9-22.

Gogolin, I. (1994). Der monolinguale Habitus der multilingualen Schule. Münster: Waxmann.

Gogolin, I. (2010). Stichwort. Mehrsprachigkeit. Zeitschrift für Erziehungswissenschaft, 13, 529-547.

Gogolin, I. (2017). Ist Mehrsprachigkeit gut oder schlecht? Zeitschrift für Grundschulforschung, 10, $102-109$.

Gogolin, I., \& Krüger-Potratz, M. (2010). Einführung in die Interkulturelle Pädagogik. Stuttgart: UTB.

Gogolin, I., \& Neumann, A. (1997). Großstadt-Grundschule. Sprachliche und kulturelle Pluralität als Bedingung der Grundschularbeit. Münster: Waxmann.

Hachfeld, A. (2013). Kulturelle Überzeugungen und professionelle Kompetenz von Lehrenden im Umgang mit kultureller Heterogenität im Klassenzimmer. Dissertation. Berlin: FU.

Hammer, S., Fischer, N., \& Koch-Priewe, B. (2016). Überzeugungen von Lehramtsstudierenden zu Mehrsprachigkeit in der Schule. Die Deutsche Schule, 13, 147-171.

Hellmich, F., \& Görel, G. (2014). Erklärungsfaktoren für Einstellungen von Lehrerinnen und Lehrern zum inklusiven Unterricht in der Grundschule. Zeitschrift für Bildungsforschung, 4, 227-240.

Hummrich, M., \& Terstegen, S. (2020). Migration. Bd. 4. Wiesbaden: Springer.

Huxel, K. (2016). die Sprachen, die tauchen einfach jetzt öfter auf im Unterricht. Die Deutsche Schule, Beiheft, 13, 175-186.

Kratzmann, J., Jahreiß, S., Frank, M., Ertanir, B., \& Sachse, S. (2017). Einstellungen pädagogischer Fachkräfte in Kindertageseinrichtungen zur Mehrsprachigkeit. Zeitschrift für Erziehungswissenschaft, 20, 237-258.

Krumm, H.-J. (2009). Die Bedeutung der Mehrsprachigkeit in den Identitätskonzepten von Migrantinnen und Migranten. In I. Gogolin \& U. Neumann (Hrsg.), Streitfall Zweisprachigkeit - the bilingualism controversy (S. 233-248). Wiesbaden: VS.

Kunter, M., Kleickmann, T., Klusmann, U., \& Richter, D. (2011). Die Entwicklung professioneller Kompetenz von Lehrkräften. In M. Kunter, J. Baumert \& W. Blum (Hrsg.), Professionelle Kompetenz von Lehrkräften (S. 55-68). Münster: Waxmann.

Lange, S. (2017). Bildungsmaterialien im Globalen Lernen. In G. Lang-Wojtasik \& U. Klemm (Hrsg.), Handlexikon Globales Lernen (2. Aufl. S. 122-125). Münster: Klemm und Oelschläger.

Liegmann, A. B. (2012). Durchlässigkeit im Schulsystem - eine Frage der Einstellung? Berufsbezogene Überzeugungen zum Schulformwechsel. Zeitschrift für Bildungsforschung, 2, 131-149. 
Maak, D., Brede, J.R., \& Born, S. (2015). Einstellungen von Lehramtsstudierenden zu Mehrsprachigkeit erheben. In H. Rösch \& J. Webersik (Hrsg.), Deutsch als Zweitsprache (S. 263-283). Stuttgart: Klett.

Mecheril, P. (Hrsg.). (2016). Handbuch Migrationspädagogik. Weinheim: Beltz.

Meyer, M., \& Prediger, S. (2011). Vom Nutzen der Erstsprache beim Mathematiklernen. In S. Prediger \& E. Özdil (Hrsg.), Mathematiklernen unter Bedingungen der Mehrsprachigkeit (S. 185-204). Münster: Waxmann.

Nohl, A.-M. (2014). Konzepte interkultureller Pädagogik. Bad Heilbrunn: Klinkhardt.

Oser, F., \& Blömeke, S. (2012). Überzeugungen von Lehrpersonen. Zeitschrift für Pädagogik, 58, 415-421.

Ottley, J. R., Piasta, S. B., Mauck, S. A., O’Connell, A., Weber-Mayrer, M., \& Justice, L. M. (2015). The nature and extent of change in early childhood educators' language and literacy knowledge and beliefs. Teaching and Teacher Education, 52, 47-55.

Özer, D., Nalbant, S., Ağlamıs,, E., Baran, F., Kaya Samut, P., Aktop, A., et al. (2013). Physical education teachers' attitudes towards children with intellectual disability. Journal of Intellectual Disability Research (JIDR), 57, 1001-1013.

Pajares, M. F. (1992). Teachers' beliefs and educational research: cleaning up a messy construct. Review of Educational Research, 62, 307-332.

Pohlmann-Rother, S. \& Lange, S. D. (2020). Mehrsprachigkeit in der Lehrkräftebildung: Empirische Ergebnisse zu den Überzeugungen von Grundschullehrkräften und Implikationen für die Kooperation von KiTa und Grundschule. In S. Pohlmann-Rother, S. D. Lange \& U. Franz (Hrsg.), Kooperation von KiTa und Grundschule. Digitalisierung, Inklusion und Mehrsprachigkeit - Aktuelle Herausforderungen beim Übergang bewältigen (Bd. 2, S. 5-44). Köln: Carl Link.

Putjata, G., Olfert, H., \& Romano, S. (2016). Mehrsprachigkeit als Kapital - Möglichkeiten und Grenzen des Moduls „Deutsch für Schülerinnen und Schüler mit Zuwanderungsgeschichte“ in NordrheinWestfalen. Österreichischer Verband für Deutsch als Fremd- und Zweitsprache (ÖDaF), 32, 34-44.

Rauch, D. (2019). Mehrsprachigkeit - ein Problem? Zusammenhänge zwischen L1-Nutzung und schulisch relevanten Kompetenzen auf Basis von PISA 2012 Daten. Zeitschrift für Erziehungswissenschaft, 22, $125-142$.

Reusser, K., Pauli, C., \& Elmer, A. (2011). Berufsbezogene Überzeugungen von Lehrerinnen und Lehrern. In E. Terhart, H. Bennewitz \& M. Rothland (Hrsg.), Handbuch der Forschung zum Lehrerberuf (S. 642-661). Münster: Waxmann.

Schorch, G. (2007). Studienbuch Grundschulpädagogik. Bad Heilbrunn: Julius Klinkhardt.

Skott, J. (2015). The promises, problems, and prospects of research on teachers' beliefs. In H. Fives (Hrsg.), International handbook of research on teachers' beliefs (S. 13-29). NY: Routledge.

Steinbach, A. (2017). „Also in meiner Klasse wird natürlich Deutsch gesprochen“ - kritische Anfragen an institutionalisierte Handlungsroutinen im Kontext von Mehrsprachigkeit. Zeitschrift für Grundschulforschung, 10, 75-87.

Tachtsoglou, S., \& König, J. (2017). Der Einfluss universitärer Lerngelegenheiten auf das pädagogische Wissen von Lehramtsstudierenden. Zeitschrift für Bildungsforschung, 7, 291-310.

Tracy, R. (2014). Mehrsprachigkeit: Vom Störfall zum Glücksfall. In M. Krifka, J. Blaszczak, A. Leßmöllmann, A. Meinunger, B. Stiebels \& R. Tracy, et al. (Hrsg.), Das mehrsprachige Klassenzimmer. Berlin: Springer VS.

Trautmann, M. (2005). Überzeugungen vom Englischlernen. Zeitschrift für Erziehungswissenschaft, 8, 38-52.

Willis, G. B. (2005). Cognitive interviewing. A tool for improving questionnaire design. New Delhi: SAGE.

Wischmeier, I. (2012). „Teachers’ Beliefs“: Überzeugungen von (Grundschul-)Lehrkräften über Schüler und Schülerinnen mit Migrationshintergrund. In W. Wiater \& D. Manschke (Hrsg.), Verstehen und Kultur (S. 167-189). Wiesbaden: Springer VS. 